\author{
Vladimir Zavialov, Viktor Bodrov, Taras M isyura, Nataliya Popova, \\ Yuliya Zaporozhets and Vadim Dekanskiy
}

\title{
DEVELOPMENT OF MATHEMATICAL MODELS OF EXTERN AL MASS EXCHANGE UNDER CONDITIONS OF VIBROEXTRACTION FROM VEGETABLE RAW MATERIALS
}

\author{
$\mathrm{N}$ ational University of Food Technologies, \\ 68, Volodymyrska str., 01601 Kiev-33, Ukraine; pandus@i.ua
}

Received: October 07, 2013/ Revised: October 22, 2013 / Accepted: February 02, 2014

(C) Zavialov V., Borov V., Misyura T., Popova N., Zaporozhets Y., Dekanskiy V., 2015

\begin{abstract}
We present results of development of mathematical models of external mass exchange in extraction of desired products from vegetable raw materials and mathematical models for the determination of the minimum time of achievement of the equilibrium state of the system and the current concentration of a soluble substance for their using in the optimization of operation of solid-phase extraction equipment.
\end{abstract}

Keywords: mathematical models, mass transfer, diffusion, vibroextraction, concentration, minimum time.

\section{Introduction}

The design of extraction equipment was always connected with the search of new methods and forms of providing the efficient interaction of phases, which are determined by the hydrodynamics created by working elements of the apparatus. Despite the range of diverse problems of individual stages of design, there exist general approaches and criteria of determination of the process efficiency of the designed apparatus. Among these, first of all, we should mention mass-exchange characteristics that are system-used in all stages of design of extractors: determination of the working volume of the apparatus, power of the drive, the design of working mixing and, sometimes, transporting devices, which set a certain hydrodynamic structure of the working flow in the apparatus.

The use of traditional methods for the determination of the intensity of external mass exchange based on the consideration of elementary processes for bodies of the simplest geometric shape under conditions of constant convection beyond the body simplifies too much the determination of the identity of theoretical and experimental data, in particular, in the evaluation of the kinetic coefficients. On the other hand, the consideration for all the factors that influence the process (hydrodynamics of the extractant flow around the body, velocity field, and extractant concentrations around a particle, geometric features of a particle, its diffusion features, etc.) complicates the choice of the method for the determination of mass-exchange characteristics that are most important for extraction.

The authors put forward the urgent problem of development of new experimental express methods for the determination of mass-exchange characteristics of extraction equipment of different modifications. In our opinion, the method that uses the process of interaction of a system of solid bodies with liquid, e.g., the process of dissolution of salts in the liquid medium by the diffusion mechanism (without precipitation of the solid phase, evolution of the gas phase, and chemical transformations), deserves attention. The results of this method take into account only external mass exchange, the efficiency of which is provided by the perfection of the design of the extractor and its individual elements, but do not take into account internal diffusion movement of the desired component inside the solid phase, which is determined by the technological conditions of the process (temperature, particle size, elasticity of particles, water duty, etc.).

\section{Experimental}

Previously [1] we used general approaches of P. Loboda [2] for the study of the influence of lowfrequency mechanical vibrations of a vibromixing device on the rate of dissolution of model specimens of substances with the diffusion type of dissolution, namely, aluminum sulfate crystalline hydrate $\mathrm{Al}_{2}\left(\mathrm{SO}_{4}\right)_{3} \cdot 18 \mathrm{H}_{2} \mathrm{O}$, and now, extending these investigations, we are using them for the condition of periodic and continuous extraction of desired components from vegetable raw 
materials with the aim to develop mathematical models for other variants of saturation of the working medium of an extractor by the dissolved experimental substance.

Investigations were performed with the use of model specimens of aluminum sulfate of specified cylindrical shape (with the diameter of $14 \cdot 10^{-3} \mathrm{~m}$ and the height of $22 \cdot 10^{-3} \mathrm{~m}$ ) with ends protected with a special coating by the technique described in [1] on laboratory periodic and continuous vibroextractors according to the schemes shown in Figs. 1 and 2, respectively.

For instance, in the thermostatted cylindrical body (1) of the periodic vibroextractor (Fig. 1) $0.31 \mathrm{~m}$ in diameter and $0.4 \mathrm{~m}$ in height, a vibromixing system consisting of a flexible container (7) of special design [3], fixed on a sieve support (8), and connected by a rod (5) through the upper perforated disk (6) with a vibrodrive (4) is located. Experimental specimens (10) intended for dissolution were fixed in a holder (11) of a bar (12) and together with it were dipped into the specified point of measurement. The specified coordinates of the point of measurement were provided by the adjusting device (2) with rulers (3) in the radial direction $R$ from the axis of the apparatus with a step of $20 \cdot 10^{-3} \mathrm{~m}$ and in the vertical direction $h$ with a step of $50 \cdot 10^{-3} \mathrm{~m}$. The height of the column of the extractant $H$ remained constant and equal to $0.180 \mathrm{~m}$ for all water duties. Investigations were performed by using leaf tea raw material, which was charged into the perforated flexible container (7) with water duties (10:1)-(30:1) with the step of $(10: 1)$. The frequency of vibrations of the vibrosystem was varied from 3 to $9 \mathrm{~Hz}$ at the fixed amplitude of $20 \cdot 10^{-3} \mathrm{~m}$.

The experimental continuous vibroextractor (Fig. 2) was made in the form of a cylindrical shell (1) with the diameter $D=0.3 \mathrm{~m}$ and the height $H=1.5 \mathrm{~m}$ from organic glass and stainless steel. Inside the shell, vibromixing devices (7) of special design [4] consisting of a perforated disk with a board along the periphery, transporting elements and filtering elements (8) are located. The vibromixing devices (7) are alternately fixed on vertical rods (5) with the possibility of executing longitudinal reciprocal movements (through the vibrodrive 11) with the frequency of 1 to $10 \mathrm{~Hz}$ and the amplitude of 5, 10, and $15 \cdot 10^{-3} \mathrm{~m}$ (discretely). Experimental specimens intended for dissolution (10) were fixed in the holder of a bar (4) and were dipped through a guide glass pipe (3) into the specified point of measurement. The height of the extractant column $H$ in the apparatus remained constant for all water duties. Experiments were carried out by using cone hop (solid phase), which was charged under the lower transporting device with water duties of $(10: 1)$ to (30:1) with the step of (10:1). Under the action of the energy of low-frequency mechanical vibrations of the vibromixing system on the two-phase system, it separated into a solvent cake and extract, as described in [5].
For the periodic and the continuous process, we used portable water at the temperature of $293 \mathrm{~K}$ as an extractant; the specified dissolution time (dwell time) of experimental specimens in it was $60 \mathrm{~s}$, and the masses of the specimens before and after dissolution were determined. It should be noted that the dissolution of chemically homogeneous (individual) substances proceeds on the external surface, and, during extraction of components from the vegetable raw material, the external surface of their particles has an irregular structure, and the dissolution process of components propagates into their internal layers. It is clear that the evaluation of the efficiency of external mass exchange by the given procedure has a certain error, which is relatively insignificant in practical application in the design of the apparatus and optimization of the process in it. The dissolution rate of the chemically homogeneous substance depends on the resistance to diffusion transfer from the internal layers of particles of the raw material to the interface and resistance to dissolution of the substance on the surface of particles and to removal of the substance from the surface of the particle into the bulk of the solvent. Fig. 3 illustrates the concentration distribution of a component near the surface of dissolution. Since, in this case, the stage of dissolution is limiting, the concentration of the substance on the surface of the specimen (particle) is the saturation concentration, and the mass transfer coefficient is practically equal to the dissolution coefficient $K_{m}$, which is determined from the main differential equation of mass transfer

$$
d m=K_{m} \cdot F(t) \cdot\left(C_{\mathrm{H}}-C(t)\right) d t
$$

where $m$ is the mass of the dissolved substance of the specimen, $C_{\mathrm{H}}$ and $C(t)$ are the saturation concentration of the substance in the boundary layer of the specimen and the current concentration of the substance in the main mass of the solvent, respectively, $F(t)$ is the current surface of dissolution of the specimen, and $t$ is the current time of dissolution.

Taking into account the aforesaid [1], we obtained the following equation for the determination of the dissolution coefficient $K_{m}$ for the condition of dissolution when the mass of the specimen does not exceed its mass under the conditions of the equilibrium state of the specimen-solvent system, i.e., $G_{0}<n^{*}$ or $G_{0}<C^{*} \cdot V$ :

$$
\begin{gathered}
K_{m}=\frac{2 \cdot V \cdot \sqrt{G_{0}}}{F(t) \cdot p \cdot\left(t-t_{0}-\Delta t\right)} . \\
\left(\operatorname{arctg} \frac{\sqrt{G_{0}-\theta / 2}}{p}-\operatorname{arctg} \frac{\sqrt{G_{V}(t)+\theta / 2}}{p}\right)
\end{gathered}
$$


where $G_{0}$ and $n^{*}$ are the initial mass of the specimen and its mass under the conditions of equilibrium of the system, respectively, $C^{*}$ is the equilibrium concentration of the substance, $V$ is the working volume of the apparatus, $F(t)$ is the current area of the surface of the specimen, $\theta$ is the change in the mass of the specimen for the time from the moment of dipping into the working point and to

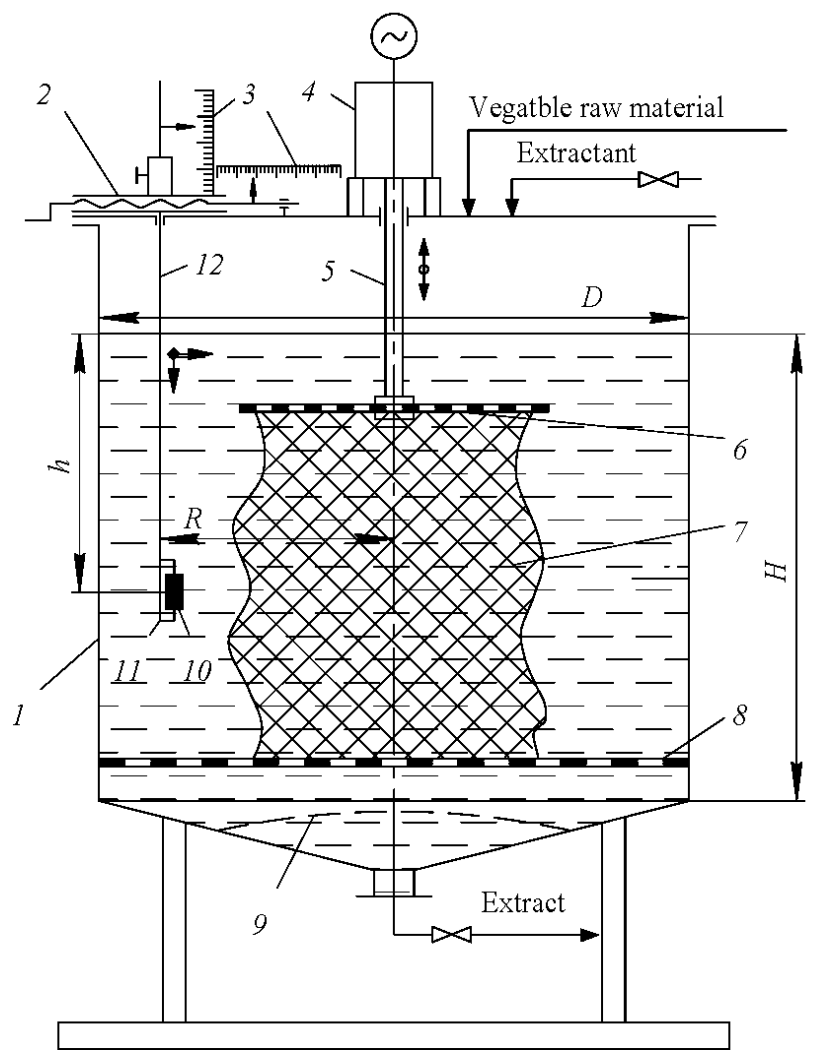

Fig. 1. Scheme of an experimental periodic vibroextractor: body of the apparatus (1); adjusting device (2); rulers (3); vibrodrive (4); rod (5); perforated disk (6); flexible container

(7); sieve support (8); mesh barrier (9); experimental specimen (10); holder of the specimen (11) and bar (12)

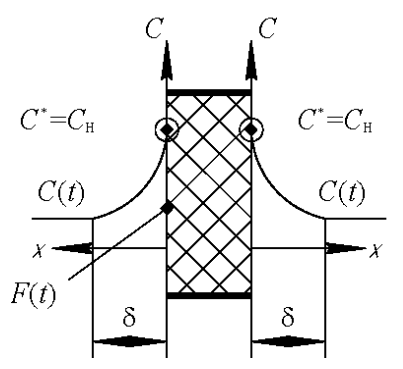

the moment of removal from it (change in the mass for the time $\left(\Delta \tau_{1}+\Delta \tau_{2}\right)$, Fig.4), $G_{V}(t)$ is the measured mass of the specimen removed from the reactor, and $p=\sqrt{C^{*} \cdot V-G_{0}}$ is the redesignation of the complex of magnitudes [1].

The scheme shown in Fig. 4 illustrates the time characteristics of the experimental measurement.

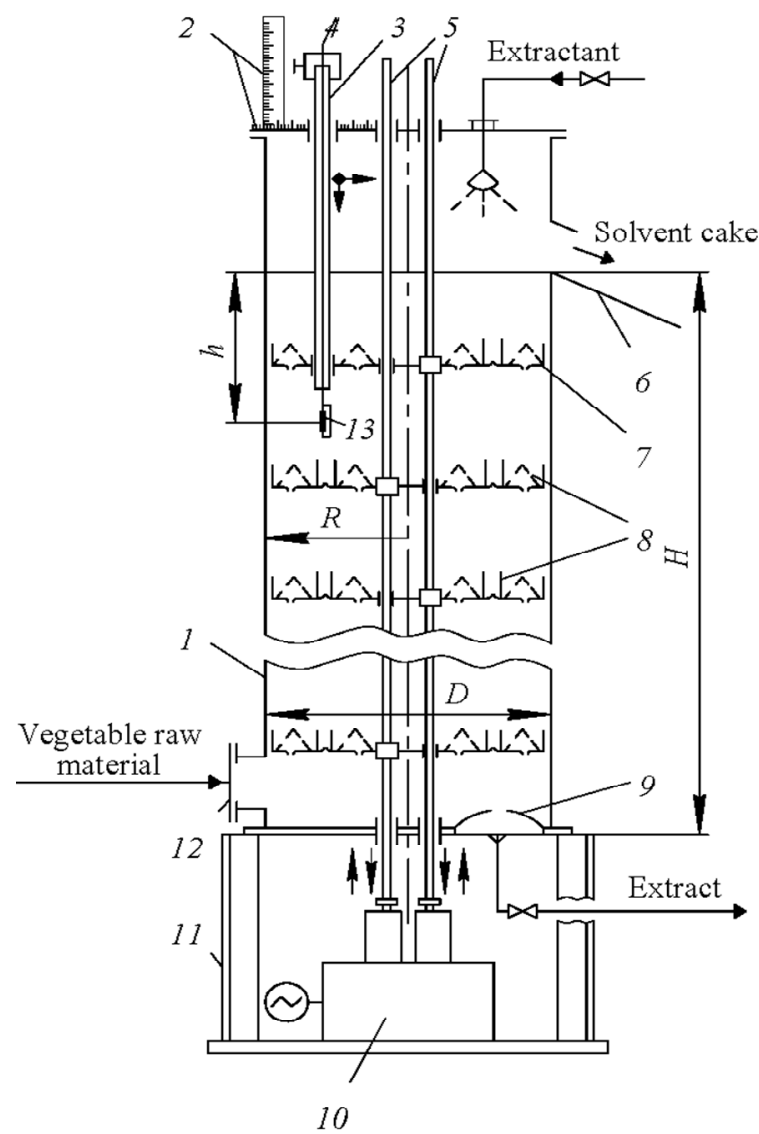

Fig. 2. Scheme of an experimental continuous vibroextractor: body (1); rulers (2); guide glass pipe (3); bar (4); rods (5); tray (6); vibromixing device (plate) (7); transporting and filtering element (8); mesh barrier (9); vibrodrive (10); frame (11); connecting branch pipe with a charging device (12) and specimen (13)

Fig. 3. Scheme of concentration distribution of the dissolved substance near the surface an experimental specimen: $F(t)$ - the varying surface of dissolution of the specimen; $C(t)$ - the current concentration of the substance in the main working flow of the extractant; $C^{*}$ and $C_{\mathrm{H}}$ - the equilibrium concentration and the saturation concentration of the substance on the surface of the specimen, respectively; $\delta$ - the thickness of the diffusion layer 


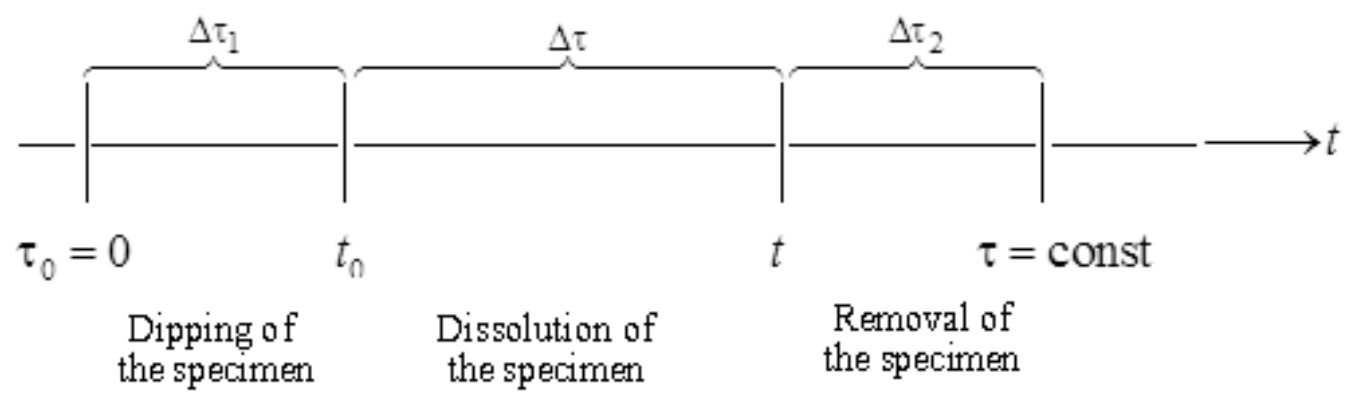

Fig. 4. Scheme of the distribution of the dwell time of a specimen in the working volume of the apparatus: $\tau_{0}$ - the contact time of the specimen with the surface of the extractant in the working volume of the apparatus;

$t_{0}$ and $t-$ the initial time and final time of the dwell of the specimen at the specified point of measurement, respectively; $\Delta \tau_{1}, \Delta \tau$, and $\Delta \tau_{2}$ - the duration of dipping of the specimen, duration of holding at the point of measurement, and duration of removal from the working volume of the apparatus, respectively: $\Delta \tau_{1}=\left(t_{0}-\tau_{0}\right) ; \Delta \tau=\left(t-t_{0}\right) ; \Delta \tau_{2}=(\tau-t) ; \tau-$ the total time of contact of the specimen with the extractant in the working volume of the apparatus $\tau=\left(\Delta \tau_{1}+\Delta \tau+\Delta \tau_{2}\right)$

The next stage of consideration of the dissolution conditions of the experimental specimen of aluminum sulfate is the case where its initial mass $G_{0}$ exceeds the current equilibrium mass of the substance $n^{*}$, i.e., $G_{0}>n^{*}$ or $G_{0}>C^{*} \cdot V$ (the second condition).

For this purpose, we perform the corresponding transformations of Eq. (21) from [1], which describes the change in the mass of a specimen with time $\Delta \tau$.

$$
\left\{\begin{array}{l}
\frac{d m(t)}{d t}=\omega \cdot \sqrt{a-m(t)} \cdot(b-m(t)) . \\
m\left(t_{0}\right)=0,
\end{array}\right.
$$

where $\omega=\frac{K_{m} \cdot F(t)}{V \cdot \sqrt{G_{0}}} ; \quad a=G_{0}-\frac{\theta}{2} ; \quad b=m^{*}=n^{*}-\frac{\theta}{2}$.

To solve Eq. (3), we use the method of separation of variables, according to which the last equation is represented in the form:

$$
\frac{d m(t)}{\sqrt{a-m(t)} \cdot(b-m)}=\omega \cdot d t
$$

where $m$ is the mass of the substance dissolved for the time $\Delta \tau=\left(t-t_{0}\right)$.

Integrating the last equation, we get

$$
\Delta=\int_{t_{0}}^{t} \frac{d m}{\sqrt{a-m} \cdot(b-m)}=\alpha \cdot\left(t-t_{0}\right)
$$

Let us first find the indefinite integral of the lefthand side of the latter equation. For this purpose, we perform the replacement of the variables, namely, $y=a-m, d y=-d m$, and obtain:

$$
\begin{gathered}
\Delta=\int \frac{d m}{\sqrt{a-m} \cdot(b-m)}= \\
=-\int \frac{d y}{\sqrt{y \cdot(b-a+y)}}=-2 \cdot \int \frac{d(\sqrt{y})}{b-a+y} .
\end{gathered}
$$

The next replacement of the variables $z=\sqrt{y}$ and $y=z^{2}$ enables us to get:

$$
\Delta=-2 \cdot \int \frac{d z}{b-a+z^{2}}=-2 \cdot \int \frac{d z}{z^{2}-(a-b)} .
$$

To obtain a tabulated (standard) integral, we perform the last replacement of the variables:

$$
\beta=\sqrt{a-b} ; \quad a-b=\beta^{2}
$$

Note: this replacement of the variables will be true on condition that $G_{0}>n^{*}$.

Let us continue transformations as follows:

$$
\begin{gathered}
\Delta=-2 \cdot \int \frac{d z}{z^{2}-(a-b)}=-2 \cdot \int \frac{d z}{z^{2}-\beta^{2}}= \\
=-2 \cdot \int \frac{d z}{(z-\beta) \cdot(z+\beta)}= \\
=-2 \cdot \int \frac{1}{(-2 \cdot \beta)} \cdot\left[\frac{1}{z+\beta}-\frac{1}{z-\beta}\right] d z= \\
=\frac{1}{\beta} \cdot\left(\int \frac{d z}{z+\beta}-\int \frac{d z}{z-\beta}\right)= \\
=\frac{1}{\beta} \cdot[\ln (z+\beta)-\ln (z-\beta)]=\frac{1}{\beta} \cdot \ln \left[\frac{z+\beta}{z-\beta}\right]= \\
=\frac{1}{\beta} \cdot \ln \left[\frac{\sqrt{a-m}+\beta}{\sqrt{a-m}-\beta}\right] .
\end{gathered}
$$


Thus, in view of the last result, with regard for Eq. (3), Eq. (4) has the form:

$$
\begin{gathered}
\Delta=\int_{t_{0}}^{t} \frac{d m}{\sqrt{a-m}(b-m)}=\frac{1}{\beta} \cdot \ln \left(\frac{\sqrt{a-m}+\beta}{\sqrt{a-m}-\beta}\right)_{t_{0}}^{t}= \\
=\frac{1}{\beta} \cdot\left\{\ln \left[\frac{\sqrt{a-m(t)}+\beta}{\sqrt{a-m(t)}-\beta}\right]-\ln \left[\frac{\sqrt{a-m\left(t_{0}\right)}+\beta}{\sqrt{a-m\left(t_{0}\right)}-\beta}\right]\right\}= \\
=\alpha\left(t-t_{0}\right)=\alpha \cdot \Delta \tau .
\end{gathered}
$$

Let us determine $m(t)$ from the last equation. For this purpose, we reduce Eq. (5) to the form:

$$
\begin{gathered}
\ln \left[\frac{\sqrt{a-m(t)}+\beta}{\sqrt{a-m(t)}-\beta}\right]= \\
=\alpha \cdot \beta\left(t-t_{0}\right)+\ln \left[\frac{\sqrt{a}+\beta}{\sqrt{a}-\beta}\right]
\end{gathered}
$$

whence we obtain:

$$
\frac{\sqrt{a-m(t)}+\beta}{\sqrt{a-m(t)}-\beta}=j
$$

where $j=e^{\alpha \cdot \beta \cdot\left(t-t_{0}\right)} \cdot\left(\frac{\sqrt{a}+\beta}{\sqrt{a}-\beta}\right)$.

Multiplying the left-hand and the right-hand side of Eq. (6) by $(\sqrt{a-m(t)}-\beta)$, we get $\sqrt{a-m(t)}+\beta=$ $=j \cdot(\sqrt{a-m(t)}-\beta)$ or $\quad \sqrt{a-m(t)} \cdot(1-j)=-\beta \cdot(1+j)$. Dividing the last equation by $(1-j)$, we obtain:

$$
\sqrt{a-m(t)}=-\beta \cdot \frac{(1+j)}{(1-j)}=\beta \cdot \frac{(j+1)}{(j-1)}
$$

By squaring the left-hand and the right-hand side of the last equality, we get

$$
\begin{aligned}
& a-m(t)=\beta^{2} \cdot\left(\frac{j+1}{j-1}\right)^{2} \\
& \text { whence } \quad m(t)=a-\beta^{2} \cdot\left(\frac{j+1}{j-1}\right)^{2}
\end{aligned}
$$

Note: taking into account that

we have:

$$
e^{\alpha \cdot \beta \cdot\left(t-t_{0}\right)} \cdot\left(\frac{\sqrt{a}+\beta}{\sqrt{a}-\beta}\right)=j,
$$

$$
j=e^{\alpha \cdot \beta \cdot\left(t-t_{0}\right)} \cdot\left(\frac{\sqrt{a}+\beta}{\sqrt{a}-\beta}\right)>\left(\frac{\sqrt{a}+\beta}{\sqrt{a}-\beta}\right)>1
$$

The last inequality is true, because $(\sqrt{a}+\beta)>(\sqrt{a}-\beta)$, and, thus, $2 \cdot \beta>0 ;$ Therefore, $(\sqrt{a}-\beta)>0$. This note justifies the validity of the last algebraic transformations.
To determine finally the mass of the specimen $m(t)$, we modify the expression:

$$
\frac{j+1}{j-1}=\frac{e^{\alpha \cdot \beta \cdot\left(t-t_{0}\right)} \cdot \frac{\sqrt{a}+\beta}{\sqrt{a}-\beta}+1}{e^{\alpha \cdot \beta \cdot\left(t-t_{0}\right)} \cdot \frac{\sqrt{a}+\beta}{\sqrt{a}-\beta}-1}
$$

Taking into account the validity of the transformations

$$
\begin{aligned}
& \frac{\sqrt{a}+\beta}{\sqrt{a}-\beta}=\frac{\sqrt{a}+\sqrt{a-\beta}}{\sqrt{a}-\sqrt{a-\beta}}=\frac{(\sqrt{a}+\sqrt{a-\beta})^{2}}{a-(a-b)}= \\
= & \frac{(\sqrt{a}+\sqrt{a-b})^{2}}{b}=\frac{e^{\alpha \cdot \beta \cdot\left(t-t_{0}\right)} \cdot(\sqrt{a}+\sqrt{a-\beta})^{2}+b}{e^{\alpha \cdot \beta \cdot\left(t-t_{0}\right)} \cdot(\sqrt{a}+\sqrt{a-\beta})^{2}-b}
\end{aligned}
$$

according to Eq. (7) and in view of $a=G_{0}-\frac{\theta}{2}, b=m^{*}$,

$$
\beta=\sqrt{a-b}=\sqrt{G_{0}-m^{*}-\frac{\theta}{2}} \text { та } \alpha=\frac{K_{m} \cdot F_{0}}{V \cdot \sqrt{G_{0}}},
$$

we finally find the following equation for the calculation of $m(t)$ :

$$
m(t)=G_{0}-\frac{\theta}{2}-\left(G_{0}-m^{*}-\frac{\theta}{2}\right) .
$$

$$
\cdot\left[\frac{\left(\sqrt{G_{0}-\frac{\theta}{2}}+\sqrt{G_{0}-m^{*}-\frac{\theta}{2}}\right)^{2}+m^{*} \cdot e^{-\gamma \cdot\left(t-t_{0}\right)}}{\left(\sqrt{G_{0}-\frac{\theta}{2}}+\sqrt{G_{0}-m^{*}-\frac{\theta}{2}}\right)^{2}-m^{*} \cdot e^{-\gamma \cdot\left(t-t_{0}\right)}}\right]^{2}
$$

Where $\quad \gamma=\alpha \cdot \beta=\frac{K_{m} F_{0}}{V} \sqrt{\frac{G_{0}-m^{*}-\frac{\theta}{2}}{G_{0}}}$

Eqs. (8) and (9) describe the dynamics of the change in the mass of the dissolved substance (specimen) in the apparatus in the case where $G_{0}>n^{*}$.

To determine the dissolution coefficient $K_{m}$, which is an analog of the mass-transfer coefficient, we return to formula (5), from which we get:

$$
\frac{1}{\beta} \cdot\left[\ln \left(\frac{\sqrt{a-m(t)}+\beta}{\sqrt{a-m(t)}-\beta}\right)-\ln \left(\frac{\sqrt{a}+\beta}{\sqrt{a}-\beta}\right)\right]=\alpha\left(t-t_{0}\right) .
$$

whence we determine the parameter $\alpha$

$$
\alpha=\frac{1}{\beta\left(t-t_{0}\right)} \cdot\left\{\ln \left[\frac{\left(\frac{\sqrt{a-m(t)}+\beta}{\sqrt{a-m(t)}-\beta}\right)}{\left(\frac{\sqrt{a}+\beta}{\sqrt{a}-\beta}\right)}\right]\right\}=
$$




$$
=\frac{1}{\beta\left(t-t_{0}\right)} \cdot\left[\ln \frac{(\sqrt{a-m(t)}+\beta) \cdot(\sqrt{a}-\beta)}{(\sqrt{a-m(t)}+\beta) \cdot(\sqrt{a}+\beta)}\right]
$$

or

$$
\frac{K_{m} \cdot F(t)}{V \cdot \sqrt{G_{0}}}=\frac{\ln \left[\frac{\left(\sqrt{G_{0}-m(t)-\frac{\theta}{2}}+\sqrt{G_{0}-m^{*}-\frac{\theta}{2}}\right) \cdot\left(\sqrt{G_{0}-\frac{\theta}{2}}-\sqrt{G_{0}-m^{*}-\frac{\theta}{2}}\right)}{\left.\left(\sqrt{G_{0}-m(t)-\frac{\theta}{2}}-\sqrt{G_{0}-m^{*}-\frac{\theta}{2}}\right) \cdot\left(\sqrt{G_{0}-\frac{\theta}{2}}+\sqrt{G_{0}-m^{*}-\frac{\theta}{2}}\right)\right]}\right.}{\sqrt{G_{0}-m^{*}-\frac{\theta}{2}\left(t-t_{0}\right)}}
$$

For convenience of representation of Eq. (10), the following notation is introduced:

$$
P_{i}=\sqrt{G_{0}-(1-i) m^{*}-\frac{\theta}{2}}, \quad i=0 ; 1
$$

Then we finally have:

$$
K_{m}=\frac{V \cdot \sqrt{G_{0}}}{F(t) \cdot P_{0}\left(t-t_{0}\right)} \cdot \ln \left[\frac{\left(\sqrt{G_{0}-m(t)-\frac{\theta}{2}}+P_{0}\right)\left(P_{1}-P_{0}\right)}{\left(\sqrt{G_{0}-m(t)-\frac{\theta}{2}}-P_{0}\right)\left(P_{1}+P_{0}\right)}\right]
$$

where $m(t)$ - the mass of the dissolved substance of the specimen in the apparatus at the moment of time $t$ and $\left(G_{0}-m(t)-\frac{\theta}{2}\right)$ is the mass of the specimen after its removal from the apparatus at the moment of time $t$.

Thus, the mass of the substance of the specimen dissolved for the dwell time at the working point, i.e. for $\Delta \tau=\left(t+t_{0}\right)$, is determined from the material balance (under the taken condition of equality of the changes the mass of the specimen during its delivery to the working point $\theta_{1}$ and during its removal from it $\theta_{2}$, i.e., $\theta_{1}=\theta_{2}=\frac{\theta}{2}$ :

$$
G(t)=G_{0}-n(t)=G_{0}-\left(m(t)+\frac{\theta}{2}\right)=G_{0}-m(t)-\frac{\theta}{2}
$$

With regard for (13), Eq. (12) for the determination of the mass-transfer coefficient takes the form:

$$
K_{m}=\frac{V \cdot \sqrt{G_{0}}}{F(t) \cdot P_{0}\left(t-t_{0}\right)} \cdot \ln \left[\frac{\left(\sqrt{G(t)}+P_{0}\right)\left(P_{1}-P_{0}\right)}{\left(\sqrt{G(t)}-P_{0}\right)\left(P_{1}+P_{0}\right)}\right]
$$

For practical calculations (with an admissible error), $F(t) \approx F_{0}$.

Let us consider the last third case where the initial mass of the specimen $G_{0}$ is equal to the current equilibrium mass of the substance in the working volume of the apparatus, i.e., $G_{0}=n^{*}$ or $G_{0}=C^{*} \cdot V$ (Eq. 2).

For this purpose, we return to Eq. (5). Taking into account that in this case $a=b$ the integrand in equation (4a) is simplified and takes the form:

$$
-2 \cdot \int \frac{d z}{z^{2}}=-2\left(-\frac{1}{z}\right)=\frac{2}{z}=\frac{2}{\sqrt{y}}=\frac{2}{\sqrt{a-m}}
$$
form:

In the case of using the last expression, $\Delta$ takes the

$$
\begin{aligned}
& \int_{t_{0}}^{t} \frac{d m}{\sqrt{a-m}(a-m)}=\left.\frac{2}{\sqrt{a-m}}\right|_{t_{0}} ^{t}= \\
= & 2 \cdot\left(\frac{1}{\sqrt{a-m(t)}}-\frac{1}{\sqrt{a}}\right)=\alpha\left(t-t_{0}\right)
\end{aligned}
$$

whence we separate $m(t)$ :

$$
\begin{gathered}
\frac{1}{\sqrt{a-m(t)}}=\frac{1}{2} \alpha\left(t-t_{0}\right)+\frac{1}{\sqrt{a}} \\
{[a-m(t)]=\left(\frac{1}{2} \alpha\left(t-t_{0}\right)+\frac{1}{\sqrt{a}}\right)^{-2}}
\end{gathered}
$$

Then $m(t)=a-\left(\frac{1}{2} \alpha\left(t-t_{0}\right)+\frac{1}{\sqrt{a}}\right)^{-2}$ or

$$
m(t)=G_{0}-\frac{\theta}{2}-\left(\frac{2 \cdot \sqrt{G_{0}-\frac{\theta}{2}}}{\alpha \sqrt{G_{0}-\frac{\theta}{2}}\left(t-t_{0}\right)+2}\right)^{2}
$$

If we denote $P_{0}=\sqrt{G_{0}-m^{*}-\frac{\theta}{2}}=\sqrt{G_{0}-C^{*} V}$ and $P_{1}=\sqrt{G_{0}-\frac{\theta}{2}}$

then, finally,

$$
m(t)=G_{0}-\frac{\theta}{2}-\left(\frac{2 P_{1}}{\alpha \cdot P_{1}\left(t-t_{0}\right)+2}\right)^{2}
$$

Let us determine $K_{m}$ in this case for $G_{0}=n^{*}$.

From Eq. (15) we have

$$
\alpha=\frac{2}{\left(t-t_{0}\right)}\left(\frac{1}{\sqrt{a-m(t)}}-\frac{1}{\sqrt{a}}\right)=\frac{K_{m} \cdot F(t)}{V \cdot \sqrt{G_{0}}}
$$

Whence

$$
K_{m}=\frac{2 V \cdot \sqrt{G_{0}}}{F(t) \cdot\left(t-t_{0}\right)} \cdot\left(\frac{1}{\sqrt{G_{0}-\frac{\theta}{2}-m(t)}}-\frac{1}{\sqrt{G_{0}-\frac{\theta}{2}}}\right)
$$

In Eq. (17), we substitute the time $t$ for $\left(t_{0}-\Delta t\right)$ :

and finally get:

$$
K_{m}=\frac{2 V \cdot \sqrt{G_{0}}}{F(t) \cdot\left(t-t_{0}-\Delta t\right)} \cdot\left(\frac{1}{\sqrt{G(t-\Delta t)}}-\frac{1}{P_{1}}\right)
$$

$$
K_{m}=\frac{2 V \cdot \sqrt{G_{0}}}{F(t) \cdot\left(t-t_{0}-\Delta t\right)} \cdot\left(\frac{1}{\sqrt{G_{V}+\frac{\theta}{2}}}-\frac{1}{P_{1}}\right)
$$


This equation is true for $G_{0}=C^{*} \cdot V$.

Let us now return to Eq. (15) and substitute the time $t$ for $(t-\Delta t)$; then we get:

$$
K_{m}=\frac{V \cdot \sqrt{G_{0}}}{F(t) P_{0}\left(t-t_{0}-\Delta t\right)} \cdot \ln \left[\frac{\left(\sqrt{G_{V}(t)+\frac{\theta}{2}}+P_{0}\right)\left(P_{1}-P_{0}\right)}{\left(\sqrt{G_{V}(t)+\frac{\theta}{2}}-P_{0}\right)\left(P_{1}+P_{0}\right)}\right]
$$

This equation is true for $G_{0}>C^{*} \cdot V$.

Combining Eqs. (2), (18), and (19) we obtain a generalized computational equation for the determination of the mass-transfer coefficient $K_{m}$ for three analyzed cases of the ratio of the masses of the dissolved substance

$$
K_{m}=\frac{V \cdot \sqrt{G_{0}}}{F(t) \cdot\left(t-t_{0}-\Delta t\right)} \cdot U(t)
$$

Where

$$
U(t)=\left\{\begin{array}{l}
\frac{1}{P_{0}} \cdot \ln \left[\frac{\left(\sqrt{G_{V}(t)+\frac{\theta}{2}}+P_{0}\right)\left(P_{1}-P_{0}\right)}{\left(\sqrt{G_{V}(t)+\frac{\theta}{2}}-P_{0}\right)\left(P_{1}+P_{0}\right)}\right] ;\left(G_{0}>C^{*} \cdot V\right) \\
\frac{2}{P}\left(\operatorname{arctg}\left[\frac{P_{1}}{P}\right]-\operatorname{arctg}\left[\frac{\sqrt{G_{V}(t)+\frac{\theta}{2}}}{P}\right]\right) ;\left(G_{0}<C^{*} \cdot V\right) \\
2\left(\frac{1}{\sqrt{G_{V}(t)+\frac{\theta}{2}}}-\frac{1}{P_{1}}\right) ;\left(G_{0}=C^{*} \cdot V\right) .
\end{array}\right.
$$

The equations of system (21) contain $P_{0}=\sqrt{G_{0}-C^{*} \cdot V} ; \quad P_{1}=\sqrt{G_{0}-\frac{\theta}{2}} ; \quad P=\sqrt{C^{*} \cdot V-G_{0}} ;$ $F_{0}=2 \pi \cdot r\left(\tau_{0}\right) \cdot h ; r\left(\tau_{0}\right)$ is the radius of the specimen before dipping into the apparatus; $G_{V}(t)$ is the measured mass of the specimen after its removal from the apparatus.

The obtained system of Eqs. (20)-(21) can be used in the physical or the mathematical modeling of the periodic or continuous extraction process for the evaluation of the external mass exchange in the two-phase solid body-liquid system under different conditions of saturation of the working volume of the apparatus by the experimental substance.

In our scheduled and realized experiments (under the conditions of periodic and continuous vibroextration), the mass-transfer coefficients $K_{m}$ were determined from the equation of the obtained mathematical model (20), i.e., for the dissolution conditions where the mass of the specimen does not exceed its equilibrium value $\left(G_{0}<n^{*}\right)$.

\section{Results and Discussion}

From the obtained experimental data we determined the dependence of the mass-transfer coefficient $K_{m}$ on the effective Reynolds criterion that takes into account the hydrodynamic regime of extractant flow around the investigated specimen of the soluble substance in the volume of the apparatus $K_{m}=f\left(\operatorname{Re}_{e}\right)$.

The effective Reynolds criterion was determined from the following equation:

$$
\operatorname{Re}_{\mathrm{e}}=\frac{\omega_{i} d_{\mathrm{e}}}{v_{\mathrm{n}}}
$$

where $\omega_{i}=\omega_{0} \bar{\eta}-$ the mean integral velocity of pulsing flows (generated by the vibromixing device) over the working volume of the apparatus; $d_{\mathrm{e}}-$ the equivalent diameter of the specimen of the soluble substance; $v_{\mathrm{n}}$ the kinematic viscosity coefficient of the solvent near the surface of dissolution; $\omega_{0}=2 A f(1-\varepsilon) / \varepsilon$ - the initial velocity of pulsing flows of the working medium in the vibration period [5]; $\varepsilon$ - the part of the free cross-sectional area of the working apparatus in the zone of location of the vibromixing device; $A$ and $f$ - the amplitude and frequency of vibrations of the vibromixing system, respectively; $\bar{\eta}=1 /(1+L / D)^{m}-$ the damping coefficient of vibrations of pulsing flows of the working medium (solvent) at the distance $L$ from their source for the continuous vibroextractor; $\eta=1 /(1+R / D)^{m}$ - the damping coefficient of vibrations of pulsing flows of the working medium (solvent) at the distance $R$ from the symmetry axis of the vibrating device (for the periodic process); $D$ - the diameter of the apparatus; $m=2+0.5 \cdot 10^{6} \mu^{2}+g B-$ the exponent that takes into account the character of propagation of waves in the solid body-liquid system [6], where $\mu$ is the dynamic viscosity coefficient of the extractant, $B$ is the specific load of the apparatus with respect to the solid phase, and $g=0.006$ is the constant coefficient at $B$.

Individual results of our experiments on the determination of the influence of turbulent pulsing jets generated by overflow elements of vibrotransporting devices during continuous and periodic extraction on external mass exchange were generalized in the functional coordinates $\mathrm{Sh} / \mathrm{Sc}^{0,5}=f\left(\mathrm{Re}_{e}\right)$ by linear dependences with a varying angle of inclination and are shown, as an example, in Fig. 4. In the presented function, $\mathrm{Sh}=K_{m} \cdot d_{\mathrm{e}} / D_{\text {д }} \quad$ is the Sherwood criterion, which characterizes the ratio of the intensity of the process caused by convection to the intensity of the process caused by diffusion (under conditions of the stationary 
medium), i.e., the influence of vibrations on the rate of mass transfer; $\mathrm{Sc}=v_{\text {п }} / D_{\text {д }}$ is the Schmidt criterion, which characterizes the ratio of the intensity of diffusion of a pulse (viscosity) to the intensity of diffusion of the substance, i.e., in this case, the relative role of molecular processes of transfer of the momentum and of the transfer of the mass of the substance by diffusion; $v_{n}$ is the kinematic viscosity coefficient of the solution; $D_{\text {д }}$ is the diffusion coefficient of the specimen.

As is seen from Fig. 4, pulsing flows (line 3) for the periodic process at insignificant numbers $\operatorname{Re}_{\mathrm{e}}$ (to 80 ) have a somewhat larger efficiency of action than for the continuous process even in the absence of the solid phase. Obviously, this is affected by the design of the apparatus and by the fact that turbulization is realized in the smaller working volume. At the same time, further intensification (increase in $\mathrm{Re}_{\mathrm{e}}$ ) does not lead to an increase in external mass exchange: here, experimental points begin to locate stably horizontally, i.e., the structure of the flow becomes closer to the structure of ideal mixing.

For the continuous process, the pattern is somewhat different. The graphs (lines 1 and 2) change the slope angle depending not only on the intensity of vibrations or

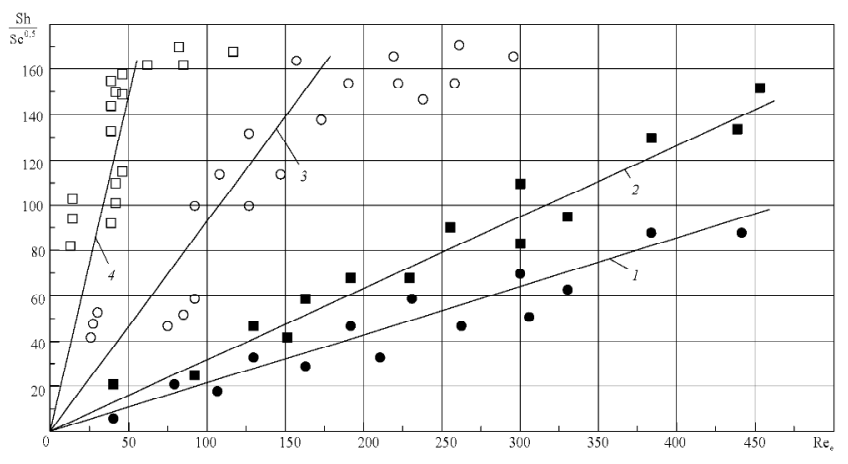

\section{Conclusions}

The obtained results and proposed methods for the determination of the level of external mass exchange can be used to determine and justify the mode in which the process of continuous or periodic vibroextraction should be carried out under production conditions and evaluate the perfection of the design of the extractor in optimization of its operation.

\section{References}

[1] Zavialov V., Lobok O., Popova N. and Bodrov V.: Nauchn. Trudy ONAKhT, 2008, 32, 88.

[2] Stabnikov B. and Loboda P.: Massoobmennye Processy Khim. Techn., Sb. Trudov. Khimiya, Leningrad 1965, 124.

[3] Zavialov V. and Popova N.: Pat. UA 25090, Publ. July 25, 2007.

[4] Zavialov V., Bodrov V. and Zaporozhets Y.: Pat. UA 27705, Publ. Nov. 12, 2007. the distance to the experimental specimen, but also the depth of interaction of pulsing flows generated simultaneously by the transporting and filtering elements of the upper and lower plates (Fig. 2, designations 7 and 8).

In this situation, the pulsing turbulent jet formed by the nozzle of the vibrating partition (plate) consists of isolated nonstationary jets in the form of vortex rings following one after another in space and time, which are generated for a half-period of vibrations of the plate. Time intervals between successive isolated jets moving in the same direction are equal to the half-period of vibrations because, during these intervals, analogous jets of opposite direction are generated. These opposing flows form stronger turbulent flows of smaller scale, which favor the renewal of the contact surface of phases at a larger rate (Fig. 4, line 2). On the other hand, this operating mode forms conditions for the emergence of longitudinal mixing, which will influence the disturbance of the continuity of the process, and, for this reason, a mode that does not cause the disturbance of the layer of the solid phase formed between plates, (i.e., a method of partitioning of the working volume of the apparatus) is desirable. In other words, for the continuous process, the mode approximated by line 1 (Fig. 5) will be reasonable and more efficient.

Fig. 5. Generalization of results of investigations of external mass exchange under conditions of periodic and continuous vibroextraction with the use of model specimens: $\bullet-$ continuous vibroextraction with a solid phase (1); - continuous vibroextraction without a solid phase (2); ○-periodic vibroextraction with a solid phase (3) and periodic vibroextraction without a solid phase (4)

[5] Zavialov V., Zaporozhets Y. and Lobok O.: Nauchn. Trudy ONAKhT, 2008, 32, 93.

[6] Stratienko O., Loboda P. and Lysyanskii V.: Izv. Vysch. Uchebn. Zaved., Ser. Pishch. Tekhnol., 1970, 5, 88.

\section{РОЗРОБЛЕННЯ МАТЕМАТИЧНИХ МОДЕЛЕЙ ЗОВНІШНЬОГО МАСООБМІНУ В УМОВАХ ВІБРОЕКСТРАГУВАННЯ ІЗ РОСЛИННОЇ СИРОВИНИ}

Анотація. Представлено результати розроблення математичних моделей зовнішнього масообміну при вилучені цүільових компонентів із рослинної сировини, а також математичних моделей для визначення мінімального часу досягнення рівноважного стану системи та поточної концентрачії розчинної речовини, - для їх застосування при оптимізації роботи твердофазової екстракиійної апаратури.

Ключові слова: математичні моделі, масовіддача, дифузія, віброекстрагування, концентрація. 